\title{
Forecast Research on the Proportion of Clean Energy Consumption in Total Energy Consumption Based on $\mathrm{GM}(1,1)$ Model
}

\author{
Wu Xin ${ }^{1, *}$, Han Pan², Yuping $\mathrm{Li}^{3}$ \\ Department of Marketing, Chongqing Technology and Business University, Nanan, Chongqing, China
}

\begin{abstract}
Clean energy can not only alleviate environmental problems but also contribute to rapid and sustainable development. The gray system is based on the sequence operator to process the original data and mine the law of change. $\operatorname{GM}(1,1)$ is a specific method of mining data, by building a $\operatorname{GM}(1,1)$ model to accumulate and generate data, the randomness of the data can be weakened and its regularity can be revealed. Here we use the $\operatorname{GM}(1,1)$ model to dynamically predict the future consumption rate of clean energy. This research not only provides data support for China's green development, but also provides suggestions for improvement based on actual conditions.
\end{abstract}

\section{Introduction}

Energy is the driving force of development, and the proportion of clean energy on the market is far lower than that of traditional energy. The over-development of some non-renewable energy not only burdens the environment but is also detrimental to subsequent sustainable development. Clean energy, that is, green energy, refers to energy that does not emit pollutants and can be directly used for production and life. It includes nuclear energy and "renewable energy." Traditional clean energy prediction methods include BP neural network model, logistic model, situation analysis, but these methods have certain defects. First of all, BP neural network prediction relies on multiple samples, while the gray prediction model can mine the deep information of small samples and is suitable for the lack of samples; the approximation and promotion capabilities of the network model are closely related to the typicality of the learning samples, and the it is difficult for sample instances to form a training set; logistic model, independent variables and Logistic probability are linear, while clean energy is affected by many factors, and there may not necessarily be a linear relationship; situation analysis method is complicated and easy due to the consideration of multiple factors. Some key factors are missing. Therefore, the $\mathrm{GM}(1,1)$ model is selected as the research method in this article, so as to more effectively use the small amount of samples available to dig out more information, better prospect the use of clean energy, and make suggestions for promoting the development of clean energy in China.

$\operatorname{GM}(1,1)^{[1-2]}$ is a forecasting model proposed by the Chinese scholar Professor Julong Deng in the 1980s, that is, a method of accumulating the original data to generate an approximate exponential law and then modeling. It is suitable for systematic forecasting of small samples and poor information. The advantage of $\operatorname{GM}(1,1)$ is that a minimum of 4 raw data can solve the uncertainty prediction problem. Using differential equations to fully excavate information, irregular raw data can be generated to obtain a regular generation sequence, which is simple and easy to verify. The successful application of $\operatorname{GM}(1,1)$ has solved a large number of practical problems in the fields of production, life, science and technology, and the application scope has been extended to the fields of finance, medicine, environment, design, energy, military, sports and so on. In recent years, a large number of researchers have conducted in-depth research on the advantages and limitations of the $\operatorname{GM}(1,1)$ algorithm, making it applicable to many fields.

\section{Practical application of the $\operatorname{GM}(1,1)$ model of the proportion of clean energy consumption in total energy consumption.}

\subsection{Model construction}

This study is taken from the raw data of the proportion of clean energy consumption in the five years from 2014 to 2018. The data comes from the 2018 China Statistical Yearbook. The specific data is shown in Figure 1. 


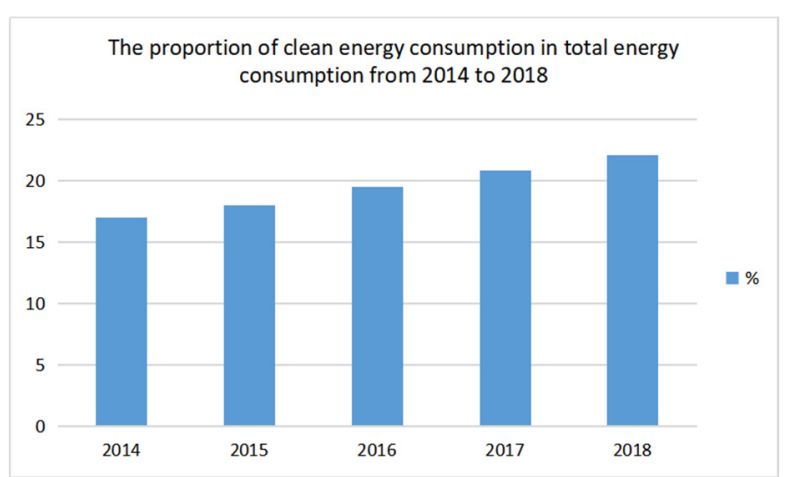

Fig 1. Data sample diagram

$$
\begin{gathered}
C^{(0)}=(17 \cdot 0,18 \cdot 0,19 \cdot 5,20.8 \cdot 22.1) \\
C^{(1)} \text { is the 1-AGO sequence of } C^{(0)}, \\
C^{(1)}=(17.000,35 \cdot 000,54 \cdot 500,75 \cdot 300,97.400)
\end{gathered}
$$

mean generation data of $C^{(1)}$ is

$$
Z^{(1)}=(26.000,44.750,64.900,86.350)
$$

The GM $(1,1)$ model based on the proportion of clean energy consumption in total energy consumption is $c^{(0)}(k)+a z^{(1)}(k)=b$, among them $\hat{a}=[a, b]^{T}=\left(B^{T} B\right)^{-1} B^{T} Y$.

$$
Y=\left[\begin{array}{c}
c^{(0)}(2) \\
c^{(0)}(3) \\
\vdots \\
c^{(0)}(n)
\end{array}\right], B=\left[\begin{array}{cc}
-z^{(1)}(2) & 1 \\
-z^{(1)}(3) & 1 \\
\vdots & \vdots \\
-z^{(1)}(n) & 1
\end{array}\right] \text {, get } \mathrm{a}=-0.07, \mathrm{~b}=16.36(4)
$$

Therefore, the time response formula of the $\operatorname{GM}(1,1)$ model in which clean energy consumption accounts for the proportion of total energy consumption is

$$
\hat{c}^{(1)}(k+1)=250.7143 e^{0.07 k}+233.7143, k=1,2, \cdots, n
$$

Get $\hat{c}^{(1)}=(17.00,35.18,54.68,75.59,98.01)$

Do a cumulative reduction, get

$$
\hat{C}^{(0)}=\left\{\hat{c}^{(0)}\right\}_{2}^{5}=(17.00,18.18,19.50,20.91,22.42)
$$

The gray prediction model software can be used to obtain a comparison chart between the original sequence and the simulated sequence, as shown in Figure 2.

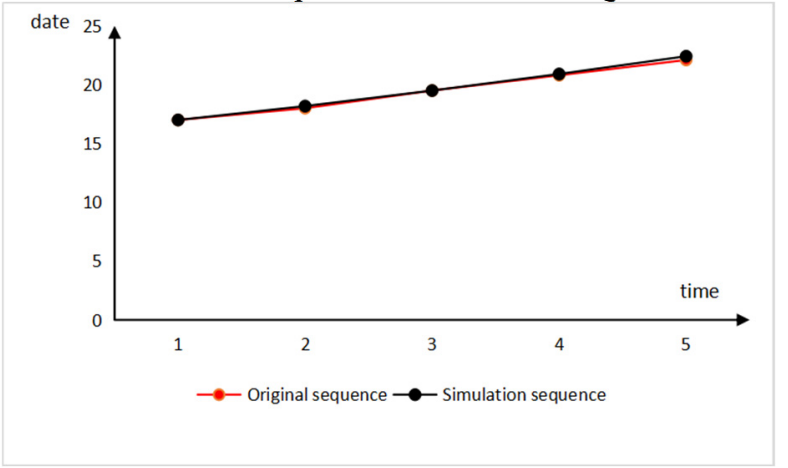

Fig 2. Alignment of original sequence and simulated sequence.

\subsection{Error check}

The original sequence is $C^{(0)}=(17.0,18 \cdot 0,19.5,20.8 .22 .1)$; The prediction sequence is

$$
\hat{C}^{(0)}=\left\{\hat{c}^{(0)}\right\}_{2}^{5}=(17.00,18.18,19.50,20.91,22.42)
$$

\subsubsection{Average relative error test}

Residual sequence:

$\varepsilon^{(0)}=(0,-0.18,0,-0.11,-0.32)$; Relative error series: $\Delta=(0,0.0100,0,0.0053,0.01448)$. Average relative error $\bar{\Delta}=0.0059$, given $\mathrm{a}=0.005$, as $\Delta=0.0059<0.01$, Accuracy is 1 level.

\subsubsection{Mean square error test}

$$
\begin{gathered}
\bar{c}=\frac{1}{5} \sum_{k=1}^{5} c^{(0)}(k)=19.48 \\
S_{1}=\sqrt{\left.\frac{1}{5} \sum_{k=1}^{5} c^{(0)}(k)-\bar{c}\right)^{2}}=1.8411 \\
\bar{\varepsilon}=\frac{1}{5} \sum_{k=1}^{5} \varepsilon(k)=-0.122 \\
S_{2}=\sqrt{\left.\frac{1}{5} \sum_{k=1}^{5} \varepsilon^{(0)}(k)-\bar{\varepsilon}\right)^{2}}=0.2692 \\
R=\frac{s_{2}}{S_{1}}=0.146<0.35
\end{gathered}
$$

Therefore, the mean square error ratio is the first level.

\subsubsection{Grey Absolute Correlation Test}

$$
\begin{aligned}
& C^{(0)}=(17.0,18.0,19.5,20.8 .22 .1) \\
& \hat{C}=(17.00,18.11,19.37,20.72,22.17)
\end{aligned}
$$

The zero image of the starting point is:

$$
\begin{gathered}
R^{0}=\left(x^{0}(1), x^{0}(2), x^{0}(3), x^{0}(4), x^{0}(5)\right) \\
=(0.000,1.000,2.500,3.800,5.100) \\
\hat{R}^{0}=\left(\hat{x}^{0}(1), \hat{x}^{0}(2), \hat{x}^{0}(3), \hat{x}^{0}(4), \hat{x}^{0}(5)\right) \\
=(0.000,1.000,2.260,3.610,5.060) \\
|s|=\left|\sum_{k=2}^{4} x^{0}(k)+\frac{1}{2} x^{0}(5)\right|=9.85 \\
|\hat{s}|=\left|\sum_{k=2}^{4} \hat{x}^{0}(k)+\frac{1}{2} \hat{x}^{0}(5)\right|=9.4 \\
|\hat{s}-s|=\left|\sum_{k=2}^{4}\left(\hat{x}^{0}(k)-x^{0}(k)\right)+\frac{1}{2}\left(\hat{x}^{0}(5)-x^{0}(5)\right)\right|=0.45(20)
\end{gathered}
$$

Thus the absolute degree of gray correlation is $\varepsilon=\frac{1+|s|+|\hat{s}|}{1+|s|+|\hat{S}|+|\hat{s}-s|}=0.9783 \quad>0.90$

So the degree of relevance is 1 level. 


\subsubsection{Small error probability test}

$$
\begin{gathered}
0.6745 S_{1}=1.24182195,|\varepsilon(1)-\hat{\varepsilon}|=0.122,|\varepsilon(2)-\hat{\varepsilon}|=-0.058 \\
|\varepsilon(3)-\hat{\varepsilon}|=0.122,|\varepsilon(4)-\hat{\varepsilon}|=0.012,|\varepsilon(5)-\hat{\varepsilon}|=-0.198(22) \\
p=p\left(|\varepsilon(k)-\hat{\varepsilon}|<0.0 .6745 S_{1}\right)=1>0.95
\end{gathered}
$$

Small probability events are classified as 1 level.

Based on the error test results and referring to the accuracy test level reference table (see Table 1), the accuracy test result of the $\operatorname{GM}(1,1)$ model for the proportion of clean energy consumption is that the relative error accuracy is level 1 , and the mean square error ratio is level 1, and the correlation The degree is one level, and the probability of small error is one level, therefore, the gray model of the proportion of clean energy consumption is established and can be used for forecasting, and the prediction accuracy is very high ${ }^{[1]}$ (See table 2 )

Table1. Accuracy inspection grade reference table

\begin{tabular}{ccccc}
\hline \multirow{2}{*}{ Accuracy class } & \multicolumn{3}{c}{ Index threshold } \\
\cline { 2 - 5 } & Relative error & Correlation & $\begin{array}{c}\text { Mean square error } \\
\text { ratio }\end{array}$ & Probability of small error \\
\hline Level 1 & 0.01 & 0.9 & 0.35 & 0.95 \\
Level 2 & 0.05 & 0.8 & 0.5 & 0.8 \\
Level 3 & 0.1 & 0.7 & 0.65 & 0.7 \\
Level 4 & 0.2 & 0.6 & 0.8 & 0.6 \\
\hline
\end{tabular}

Table2. GM $(1,1)$ model accuracy test

\begin{tabular}{ccccc}
\hline index & Relative error & Correlation & Mean square error & Probability of \\
\hline Numerical value & 0.0059 & 0.9783 & 0.146 & 1 \\
Accuracy class & Level 1 & Level 1 & Level 1 & Level 1 \\
\hline
\end{tabular}

For the prediction of the proportion of clean energy consumption in total energy consumption, the $\operatorname{GM}(1,1)$ model of the proportion of clean energy has passed the average relative error test, the mean square error test, the gray absolute correlation test and the small error probability test. The model can be used to forecast China's clean energy in the future. The following forecasts are made on the proportion of China's clean energy consumption in the next five years.

$$
\begin{gathered}
\hat{c}^{(1)}(k+1)=250.7143 e^{0.07 k}+233.7143, k=1,2, \cdots, n(23) \\
\text { and } \hat{c}^{(0)}(k+1)=\hat{c}^{(1)}(k+1)-\hat{c}^{(1)}(k)
\end{gathered}
$$

When $\mathrm{k}=5$

$$
\begin{gathered}
\hat{c}^{(1)}(5+1)=250.7143 e^{0.07 * 5}-233.7143=122.066 \\
\hat{c}^{(0)}(6)=\hat{c}^{(0)}(6)-\hat{c}^{(0)}(5)=23.72
\end{gathered}
$$

The same goes for:

$$
\text { when } \mathrm{k}=6, \hat{c}^{(0)}(7)=\hat{c}^{(0)}(7)-\hat{c}^{(0)}(6)=25.37
$$

$$
\begin{gathered}
\text { when } \mathrm{k}=7, \hat{c}^{(0)}(8)=\hat{c}^{(0)}(8)-\hat{c}^{(0)}(7)=27.14 \\
\text { when } \mathrm{k}=8, ; \hat{c}^{(0)}(9)=\hat{c}^{(0)}(9)-\hat{c}^{(0)}(8)=29.04 \\
\text { When } \mathrm{k}=9, . \hat{c}^{(0)}(10)=\hat{c}^{(0)}(10)-\hat{c}^{(0)}(9)=31.06
\end{gathered}
$$

Projected value of China's clean energy consumption as a proportion of energy consumption in the next 5 years:

$$
\begin{aligned}
& \hat{C}^{(0)}=\left(\hat{c}^{(0)}(6), \hat{c}^{(0)}(7), \hat{c}^{(0)}(8), \hat{c}^{(0)}(9), \hat{c}^{(0)}(10)\right) \\
& =(23.72,25.37,27.14,29.04,31.06)
\end{aligned}
$$

That is, in 2019, clean energy consumption accounted for $23.72 \%$ of energy consumption. In fact, clean energy consumption accounted for $23.4 \%$ of energy consumption in 2019, which is a small error from the forecast, indicating that the forecast has a high degree of credibility. In 2020, clean energy consumption will account for $25.37 \%$ of energy consumption. In 2021, clean energy consumption will account for $27.14 \%$ of energy consumption. In 2022, clean energy consumption will account for $29.04 \%$ of energy consumption. In 2023 , clean energy consumption will account for $31.06 \%$. See Table 3 .

Table3. Forecast of the proportion of clean energy consumption in energy consumption

\begin{tabular}{cccccc}
\hline years & 2019 & 2020 & 2021 & 2022 & 2023 \\
Proportion of clean energy consumption\% & 23.72 & 25.37 & 27.14 & 29.04 & 31.06 \\
\hline
\end{tabular}




\section{3 conclusion}

The GM(1,1) model, which accounts for the proportion of clean energy consumption in total energy consumption, has passed the above four tests, has high simulation accuracy, and can make relatively accurate predictions. China is a big energy consuming country, and most of the resources we currently develop and use are nonrenewable and will have a negative impact on the environment. After forecasting, we can clearly see that China's future consumption of clean energy will show an increasing trend. But the growth rate is flat, far behind the economic development speed, indicating that there is a large room for growth in the development and use of clean energy in my country. The author here makes the following suggestions:

- The state promulgated some policies to promote clean energy consumption;

- Intensify the research and development of clean energy development to ensure the efficiency and safety of use;

-Companies try to use clean energy in their manufacturing operations;

-We should also actively choose to use clean energy in our daily lives.

\section{Acknowledgments}

Project Supported by Social Science Planning Program of Chongqing (2016BS033); Project Supported by Social and Scientific Research Program of Chongqing Municipal Education Commission (17SKG096); Project Supported by Scientific and Technological Research Program of Chongqing Municipal Education Commission (KJ1600612).

\section{References}

1. Deng, J. (1989). Introduction to grey system theory. Journal of Grey Systems, 1(1),1-24.

2. Yu-Zhe Z, Chun-You W. An Improved GM $(1,1)$ Model of Integrated Optimizing Its Background Value and Initial Condition[J]. Advances in Intelligent and Soft Computing, 2010, 78.

3. Cheng M, Shi G, Xiang M . On the improvement of the parameter estimation of the grey model $\operatorname{GM}(1,1)$ and model application[J]. Communications in Statistics - Simulation and Computation, 2020, 49(5):1367-1384.

4. Huiru Z, Haoran Z, Sen G. Using GM $(1,1)$ Optimized by MFO with Rolling Mechanism to Forecast the Electricity Consumption of Inner Mongolia[J]. Applied Sciences, 2016, 6(1):20-20. 